\title{
EQUIVALENCE DE DEUX FIBRATIONS POUR LES FEUILLETAGES LOGARITHMIQUES
}

\author{
ABDELHAK KABILA
}

Abstract

The object of this work is to generalize to the germs of Pfaffian logarithmic forms, the two equivalent descriptions of the J. Milnor's fibre that is wellknown for the germs of holomorphic functions.

\section{Introduction}

Soit $f$ une fonction analytique définie au voisinage de l'origine 0 de $\mathbb{C}^{n}$. On suppose que $f(0)=0$ et que $f$ est à singularité isolée en 0.

$\varepsilon$ et $\eta$ étant des réels strictement positifs on note:

- $\varepsilon B^{2 n}$ (respectivement $D_{\eta}^{2}$ ) la boule ouverte de centre 0 et de rayon $\varepsilon$ dans $\mathbb{C}^{n}$ (respectivement le disque fermé de centre 0 et de rayon $\eta$ dans $\mathbb{C}$ ).

- $\varepsilon S^{2 n-1}$ la sphère de centre 0 et de rayon $\varepsilon$ dans $\mathbb{C}^{n}$.

- $\varepsilon K=\varepsilon S^{2 n-1} \cap f^{-1}(0)$.

Pour $\varepsilon$ et $\eta$ assez petits J. Milnor a démontré dans [2] que chacune des deux applications suivantes, $\Phi^{\prime}$ et $\Phi^{\prime \prime}$ définies par:

$$
\Phi^{\prime}: z \in \varepsilon S^{2 n-1} \backslash \varepsilon K \rightarrow \frac{f(z)}{|f(z)|} \in S^{1}
$$

et

$$
\Phi^{\prime \prime}: z \in f^{-1}\left(\partial D_{\eta}^{2}\right) \cap \varepsilon B^{2 n} \rightarrow f(z) \in \partial D_{\eta}^{2}
$$

est une fibration $C^{\infty}$ localement triviale, et qu'en plus ces deux fibrations sont équivalentes ce qui donne deux descriptions équivalentes de la fibre de J. Milnor.

Le but de ce travail est de généraliser ces résultats aux germes de formes de Pfaff logarithmiques. 


\section{Généralisation aux feuilletages logarithmiques}

Soient $f_{1}, f_{2}, \ldots, f_{m}, m$ germes de fonctions holomorphes à l'origine 0 de $\mathbb{C}^{n}$, et $\lambda_{1}, \lambda_{2}, \ldots, \lambda_{m}, m$ nombres complexes non tous nuls. Nous supposons que tous les $f_{k}$ sont premiers entre eux et que $f_{k}(0)=0$ pour tout $k$ compris entre 1 et $m$.

Désignons par:

- $\omega$ le germe en $0 \in \mathbb{C}^{n}$ de forme de Pfaff holomorphe défini par

$$
\omega=f_{1} f_{2} \ldots f_{m} \sum_{k=1}^{m} \lambda_{k} \frac{d f_{k}}{f_{k}} .
$$

- $\Omega$ la forme de Pfaff fermée suivante:

$$
\Omega=\sum_{k=1}^{m}\left[\operatorname{Im} \lambda_{k} d\left(\log \left|f_{k}\right|\right)+\operatorname{Re} \lambda_{k} d\left(\operatorname{Arg} f_{k}\right)\right]
$$

où $\operatorname{Im} *$ (respectivement $\operatorname{Re} *$ ) désigne la partie imaginaire (respectivement réelle) de $*$, et $\operatorname{Arg} f_{k}(z)$ désigne l'argument de $f_{k}(z)$ pour $z \in \mathbb{C}^{n}$.

- $\varepsilon B^{2 n}$ (respectivement $\varepsilon S^{2 n-1}$ ) désigne la boule (respectivement la sphère) de centre 0 et de rayon $\varepsilon$ dans $\mathbb{C}^{n}$.

- $\varepsilon K=\varepsilon S^{2 n-1} \cap X_{0}$, où $X_{0}$ désigne le germe d'hypersurface d'équation réduite:

$$
f_{1} f_{2} \ldots f_{m}=0
$$

- $F$ le germe, en $0 \in \mathbb{C}^{n}$, de fonction multiforme:

$$
f_{1}^{\lambda_{1}} f_{2}^{\lambda_{2}} \ldots f_{m}^{\lambda_{m}}
$$

- $\nu_{1}, \nu_{2}, \ldots, \nu_{m}$, respectivement l'ordre en $0 \in \mathbb{C}^{n}$ de $f_{1}, f_{2}, \ldots, f_{m}$.

\section{Remarque 1.}

L'extension aux germes de formes de Pfaff logarithmiques de certains résultats liées à la structure de la fibration de Milnor d'un germe de fonction holomorphe impose d'introduire l'hypothèse naturelle de non résonnance $\sum_{k=1}^{m} \lambda_{k} \nu_{k} \neq 0$.

\section{Remarque 2.}

On peut toujours, quitte à changer les $\lambda_{k}, 1 \leq k \leq m$, au départ, ce qui ne modifie pas le feuilletage, supposer que $\sum_{k=1}^{m} \lambda_{k} \nu_{k}$ est un réel 
strictement positif. Plus précisement si $\sum_{k=1}^{m} \lambda_{k} \nu_{k}$ n'est pas un réel, on pose:

$$
\rho=\sum_{k=1}^{m} \overline{\lambda_{k}} \nu_{k}
$$

et on considère la forme de Pfaff holomorphe suivante:

$$
f_{1} \cdot f_{2} \ldots f_{m} \sum_{k=1}^{m} \mu_{k} \frac{d f_{k}}{f_{k}}
$$

où

$$
\mu_{k}=\rho \lambda_{k} \text { pour tout } k \text { compris entre } 1 \text { et } m \text {. }
$$

Il est clair que le feuilletage défini par la forme de Pfaff $f_{1} \cdot f_{2} \ldots f_{m} \sum_{k=1}^{m} \mu_{k} \frac{d f_{k}}{f_{k}}$ est le même que celui défini par la forme de Pfaff $f_{1} \cdot f_{2} \ldots f_{m} \sum_{k=1}^{m} \lambda_{k} \frac{d f_{k}}{f_{k}}$.

De plus:

$$
\begin{aligned}
\sum_{k=1}^{m} \mu_{k} \nu_{k} & =\sum_{k=1}^{m} \rho \lambda_{k} \nu_{k}=\rho \sum_{k=1}^{m} \lambda_{k} \nu_{k} \\
& =\left(\sum_{k=1}^{m} \overline{\lambda_{k}} \nu_{k}\right)\left(\sum_{k=1}^{m} \lambda_{k} \nu_{k}\right)=\left|\sum_{k=1}^{m} \lambda_{k} \nu_{k}\right|^{2}
\end{aligned}
$$

qui est bien un réel strictement positif.

Sachant que les feuilles du feuilletage défini par le germe de forme de Pfaff $\omega$ (respectivement $\Omega$ ) coïncident avec les composantes connexes des "hypersurfaces de niveaux" du germe de fonction multiforme $F$ (respectivement $\operatorname{Arg} F$ ), rappelons que nous avons démontré dans [1] que pour $\varepsilon$ réel strictement positif assez petit, il existe sur $\varepsilon S^{2 n-1} \backslash \varepsilon K$ un flot qui permute les feuilles du feuilletage défini par la forme $\Omega$, ce qui est une généralisation aux germes de formes de Pfaff logarithmiques du théorème de fibration de J. Milnor bien connu pour les germes de fonctions. 
Nous nous proposons ici de démontrer le résultat suivant:

\section{Théorème.}

Il existe $\varepsilon_{0}$ et $\eta_{0}$ réels strictement positifs tel que pour tout $\varepsilon$ et pour tout $\eta$, vérifiant $0<\varepsilon \leq \varepsilon_{0}, 0<\eta \leq \eta_{0}$, il existe un $C^{\infty}$ difféomorphisme $d u$ "tube" $[|F|=\eta] \cap \varepsilon B^{2 n}$ sur $\varepsilon S^{2 n-1} \backslash \varepsilon K$ qui transforme le feuilletage défini par $\omega$ sur $[|F|=\eta] \cap \varepsilon B^{2 n}$ en le feuilletage défini par $\Omega$ sur $\varepsilon S^{2 n-1} \backslash \varepsilon K$.

\section{Démonstration:}

On utilise les mêmes techniques que dans [2]. Rappelons d'abord quelques notations.

Dans tout ce qui suit l'espace vectoriel $\mathbb{C}^{n}$ est supposé muni du produit hermitien:

$$
\begin{aligned}
& \langle,\rangle: \mathbb{C}^{n} \times \mathbb{C}^{n} \quad \longrightarrow \mathbb{C} \\
& \left(\left(a_{k}\right)_{1 \leq k \leq n},\left(b_{k}\right)_{1 \leq k \leq n}\right) \quad \longmapsto\left\langle\left(a_{k}\right)_{1 \leq k \leq n},\left(b_{k}\right)_{1 \leq k \leq n}\right\rangle=\sum_{k=1}^{n} a_{k} \overline{b_{k}}
\end{aligned}
$$

et de la norme induite:

$$
\begin{aligned}
& \|\|: \mathbb{C}^{n} \quad \longrightarrow \mathbb{R}^{+} \\
& \left(a_{k}\right)_{1 \leq k \leq n} \longmapsto\left\|\left(a_{k}\right)_{1 \leq k \leq n}\right\|=\sqrt{\left\langle\left(a_{k}\right)_{1 \leq k \leq n},\left(a_{k}\right)_{1 \leq k \leq n}\right\rangle} .
\end{aligned}
$$

\section{Définition.}

Soit $\Psi:\left(\mathbb{C}^{n}, 0\right) \rightarrow\left(\mathbb{C}^{n}, 0\right)$ un germe d'application holomorphe à l'origine 0 de $\mathbb{C}^{n}$. On appelle gradient de $\Psi$ et on note grad $\Psi$ l'expression suivante:

$$
\operatorname{grad}(\Psi)=\left(\frac{\overline{\partial \Psi}}{\partial z_{1}}, \frac{\overline{\partial \Psi}}{\partial z_{2}}, \ldots, \frac{\overline{\partial \Psi}}{\partial z_{n}}\right)
$$

où $\left(z_{1}, z_{2}, \ldots, z_{n}\right)$ désignent les coordonnées de $\mathbb{C}^{n}$.

\section{Remarque 3.}

Si $P:(\mathbb{C}, 0) \rightarrow\left(\mathbb{C}^{n}, 0\right)$ est un germe, en $0 \in \mathbb{C}^{n}$, de chemin analytique alors on a:

$$
t \quad \rightarrow \quad P(t)
$$

$$
\frac{d(\Psi(P(t)))}{d t}=\left\langle\frac{d P(t)}{d t}, \operatorname{grad}(\Psi(P(t))\rangle\right.
$$




\section{Remarque 4.}

Soit $\varphi:\left(\mathbb{C}^{n}, 0\right) \rightarrow(\mathbb{C}, 0)$ un germe de fonction holomorphe à l'origine 0 de $\mathbb{C}^{n}$ alors on a:

$$
\operatorname{grad}(\varphi(z))=\overline{\varphi(z)} \operatorname{grad}(\log (\varphi(z)))
$$

où $\log (\varphi(z))$ désigne le logarithme de $\varphi(z)$ pour $z \in \mathbb{C}^{n}$.

La démonstration du théorème se fait en plusieurs étapes. Nous allons commencer par démontrer un certain nombre de propositions:

\section{Proposition 1.}

Quelque soit $z \in \mathbb{C}^{n} \backslash X_{0}$, suffisamment proche de $0 \in \mathbb{C}^{n}$, les vecteurs $z$ et $i \sum_{k=1}^{m} \overline{\lambda_{k}} \operatorname{grad} \log f_{k}(z)$ sont linéairement indépendants sur $\mathbb{R}$.

Démonstration de la Proposition 1:

Elle découle sans peine du résultat suivant:

\section{Proposition 2.}

Il existe $\varepsilon_{0}$ réel strictement positif tel que pour tout $z \in \varepsilon B^{2 n} \backslash X_{0}, 0<$ $\varepsilon \leq \varepsilon_{0}$ les vecteurs $z$ et $\sum_{k=1}^{m} \overline{\lambda_{k}} \operatorname{grad} \log f_{k}(z)$ sont ou bien linéairement indépendants sur $\mathbb{C}$, ou bien on a $\sum_{k=1}^{m} \overline{\lambda_{k}} \operatorname{grad} \log f_{k}(z)=\alpha z$ avec $\alpha \in$ $\mathbb{C} \backslash\{0\}$ et $|\operatorname{Arg} \alpha|<\frac{\pi}{4}$.

Démonstration de la Proposition 2:

Nous avons besoin du lemme suivant:

\section{Lemme 1.}

Supposons que $\sum_{k=1}^{p} \lambda_{k} \nu_{k}>0$ et soit $P:[0, r) \rightarrow \mathbb{C}^{n}$ un chemin analytique réel tel que:

(1) $P(0)=0$ et pour tout $t$ strictement positif $P(t) \notin X_{0}$.

(2) $\sum_{k=1}^{m} \overline{\lambda_{k}} \operatorname{grad} \log f_{k}(P(t))=\alpha(t) P(t)$ avec $\alpha(t) \in \mathbb{C}$. 
Alors il existe $r^{\prime} \leq r$ tel que pour tout $t$, vérifiant $0<t<r^{\prime}$ on a:

$$
\alpha(t) \neq 0 .
$$

Démonstration du Lemme 1:

Soit $t$ strictement positif. Pour tout $k$ compris entre 1 et $m$ on a: $f_{k}(P(t)) \neq 0$ car $P(t) \notin X_{0}$.

Ecrivons:

$$
\begin{array}{cl}
P(t)=a t^{l}+\cdots & a \in \mathbb{C}^{n} \backslash\{0\} \text { et } l=\left(l_{1}, l_{2}, \ldots, l_{n}\right) \\
& \text { avec } l_{1}, l_{2}, \ldots, l_{n} \in \mathbb{N} \backslash\{0\} \\
f_{k}(P(t))=b_{k} t^{\beta_{k}}+\cdots & b_{k} \in \mathbb{C} \backslash\{0\} .
\end{array}
$$

Posons:

$$
\Psi=\overline{f_{1}} \cdot \overline{f_{2}} \ldots \overline{f_{m}} \quad \sum_{k=1}^{m} \overline{\lambda_{k}} \operatorname{grad} \log f_{k} .
$$

Puisque $P$ est analytique on a:

$$
\begin{array}{r}
\Psi(P(t))=c t^{\gamma}+\cdots \quad c \in \mathbb{C}^{n} \backslash\{0\} \text { et } \gamma=\left(\gamma_{1}, \gamma_{2}, \ldots, \gamma_{n}\right) \\
\text { avec } \gamma_{1}, \gamma_{2}, \ldots, \gamma_{n} \in \mathbb{N} \backslash\{0\} .
\end{array}
$$

D'autre part, on a:

$$
\begin{aligned}
\Psi(P(t)) & =\overline{f_{1}}(P(t)) \overline{f_{2}}(P(t)) \ldots \overline{f_{m}}(P(t)) \alpha(t) P(t) \\
& =\alpha(t)\left(a \overline{b_{1} b_{2}} \ldots \overline{b_{m}} t^{l+\beta_{1}+\beta_{2}+\cdots+\beta_{m}}+\cdots\right)
\end{aligned}
$$

en identifiant $\beta_{k}$ avec $\left(\beta_{k}, \beta_{k}, \ldots, \beta_{k}\right) \in \mathbb{N}^{n}$ pour tout $k$ compris entre 1 et $m$.

Ce qui implique que:

$$
c t^{\gamma}+\cdots=\alpha(t) a \overline{b_{1} b_{2}} \ldots \overline{b_{m}} t^{l+\beta_{1}+\beta_{2}+\cdots+\beta_{m}}+\cdots
$$

Ecrivons:

$$
c=\left(c_{1}, c_{2}, \ldots, c_{n}\right)
$$

on a $c \in \mathbb{C}^{n} \backslash\{0\}$, donc il existe $s$ compris entre 1 et $n$ tel que $c_{s} \neq 0$. Ce qui entraine nécessairement d'après l'égalité ci-dessus que d'une part $a_{s} \neq 0$ et que d'autre part $\alpha(t) \neq 0$.

Ainsi:

$$
\alpha(t) \in \mathbb{C} \backslash\{0\} .
$$


De plus:

$$
\alpha(t)=\alpha_{0} t^{\gamma_{s}-l_{s}-\beta_{1}-\beta_{2} \ldots-\beta_{m}}(1+\cdots)
$$

avec:

$$
\alpha_{0}=\frac{c_{s}}{a_{s} \overline{b_{1} b_{2}} \ldots \overline{b_{m}}} .
$$

On cherche à montrer que $\alpha_{0}$ est un nombre réel.

D'après la Remarque 3 , on a pour tout $k$ compris entre 1 et $m$ :

$$
\frac{d f_{k}(P(t))}{d t}=\left\langle\frac{d P(t)}{d t}, \operatorname{grad} f_{k}(P(t))\right\rangle
$$

donc:

$$
\begin{aligned}
& f_{1}(P(t)) f_{2}(P(t)) \ldots f_{m}(P(t)) \sum_{k=1}^{m} \frac{\lambda_{k}}{f_{k}(P(t))} \frac{d f_{k}(P(t))}{d t} \\
= & \left\langle\frac{d P(t)}{d t}, f_{1}(P(t)) f_{2}(P(t)) \ldots f_{m}(P(t)) \sum_{k=1}^{m} \frac{\overline{\lambda_{k}}}{\overline{f_{k}(P(t))}} \operatorname{grad} f_{k}(P(t))\right\rangle .
\end{aligned}
$$

D'après la Remarque 4 , on a pour tout $k$ compris entre 1 et $m$ :

$$
\operatorname{grad} f_{k}(P(t))=\overline{f_{k}(P(t))} \operatorname{grad} \log f_{k}(P(t)) .
$$

Donc:

$$
\begin{aligned}
f_{1}(P(t)) f_{2}(P(t)) \ldots f_{m}(P(t)) \sum_{k=1}^{m} \frac{\lambda_{k}}{f_{k}(P(t))} \frac{d f_{k}(P(t))}{d t} \\
=\left\langle\frac{d P(t)}{d t}, \Psi(P(t))\right\rangle .
\end{aligned}
$$

Ainsi:

$$
\begin{aligned}
\sum_{k=1}^{m} \lambda_{k} b_{1} b_{2} \ldots b_{m} \beta_{k} t^{\beta_{1}+\beta_{2}+\cdots}+\beta_{m}-1 & +\cdots \\
= & \left\langle A t^{L}+\cdots, \alpha_{0} a \overline{b_{1} b_{2}} \ldots \overline{b_{m}} t^{\gamma}+\cdots\right\rangle .
\end{aligned}
$$

Avec:

$$
A=\left(l_{1} a_{1}, l_{2} a_{2}, \ldots, l_{m} a_{m}\right) \text { et } L=\left(l_{1}-1, l_{2}-1, \ldots, l_{m}-1\right) .
$$

Donc:

$$
\sum_{k=1}^{m} \lambda_{k} \beta_{k}=\overline{\alpha_{0}}\langle A, a\rangle
$$


or:

$$
\langle A, a\rangle=\sum_{k=1}^{m} l_{k}\left|a_{k}\right|^{2} \in \mathbb{R} .
$$

Donc:

$$
\sum_{k=1}^{m} \lambda_{k} \beta_{k}=\overline{\alpha_{0}} \sum_{k=1}^{m} l_{k}\left|a_{k}\right|^{2} .
$$

D'autre part si on désigne par $\nu_{1}, \nu_{2}, \ldots, \nu_{m}$ respectivement les ordres en $o \in \mathbb{C}^{n}$ de $f_{1}, f_{2}, \ldots, f_{m}$, on peut d'après le théorème de préparation de Weierstrass ( $\operatorname{cf}[4]$ ) écrire $f_{1}, f_{2}, \ldots, f_{m}$ sous la forme suivante:

$$
\begin{array}{cccc}
f_{1}\left(z_{1}, z_{2}, \ldots, z_{n}\right) & = & U_{1}\left(z_{1}, z_{2}, \ldots, z_{n}\right) & \left(z_{s}^{\nu_{1}}+\cdots\right) \\
f_{2}\left(z_{1}, z_{2}, \ldots, z_{n}\right) & = & U_{2}\left(z_{1}, z_{2}, \ldots, z_{n}\right) & \left(z_{s}^{\nu_{2}}+\cdots\right) \\
\vdots & & \vdots & \vdots \\
f_{m}\left(z_{1}, z_{2}, \ldots, z_{n}\right) & = & U_{m}\left(z_{1}, z_{2}, \ldots, z_{n}\right) & \left(z_{s}^{\nu_{m}}+\cdots\right)
\end{array}
$$

où $U_{1}, U_{2}, \ldots, U_{m}$ sont des unités de l'anneau $\Theta_{\mathbb{C}^{n}, 0}$ des germes de fonctions holomorphes à l'origine 0 de $\mathbb{C}^{n}$ i.e. $U_{k}(0) \neq 0$ pour tout $k$ compris entre 1 et $m$.

Ainsi:

$$
\beta_{k}=l_{s} \nu_{k} \text { pour tout } k \text { compris entre } 1 \text { et } m \text {. }
$$

Donc l'égalité:

$$
\sum_{k=1}^{m} \lambda_{k} \beta_{k}=\overline{\alpha_{0}} \sum_{k=1}^{m} l_{k}\left|a_{k}\right|^{2}
$$

devient:

$$
l_{s}\left(\sum_{k=1}^{m} \lambda_{k} \nu_{k}\right)=\overline{\alpha_{0}}\left(\sum_{k=1}^{m} l_{k}\left|a_{k}\right|^{2}\right) .
$$

Or:

$$
\left.\sum_{k=1}^{m} \lambda_{k} \nu_{k} \in\right] 0,+\infty[
$$

ce qui entraine que $\left.\overline{\alpha_{0}} \in\right] 0,+\infty\left[\right.$ et par conséquent $\alpha_{0}$ aussi.

Visiblement:

$$
\frac{\alpha(t)}{|\alpha(t)|}=\frac{\alpha_{0} t^{\gamma_{S}-l_{S}-\beta_{1}-\beta_{2}-\cdots-\beta_{m}}(1+t[\ldots])}{\left|\alpha_{0} t^{\gamma_{S}-l_{S}-\beta_{1}-\beta_{2}-\cdots-\beta_{m}}(1+t[\ldots])\right|}
$$

tend vers 1 quand $t$ tend vers zéro.

Et le Lemme 1 est démontré.

Rappelons aussi le lemme suivant bien connu sous le nom de "lemme des petits chemins" ou encore de "lemme de sélection des courbes". 
Lemme 2 (cf. [2]).

Soit $A \subset \mathbb{R}^{P}$ un ensemble semi-analytique, et soit a un point adhérent $\grave{a} A$. Alors il existe un chemin analytique réel $P:[0, r) \rightarrow \mathbb{R}^{P}$ tel que:

(1) $P(0)=a$

(2) $P(t) \in$ A pour tout $t$ strictement positif.

Déduisons maintenant la Proposition 2 à partir des lemmes précédents. Considérons l'ensemble $A$ suivant:

$$
A=\left\{\begin{array}{cl}
z \in \mathbb{C}^{n} \backslash X_{0} \text { tel que: } & \exists \alpha \in \mathbb{C}: \sum_{k=1}^{m} \overline{\lambda_{k}} \operatorname{grad} \log f_{k}(z)=\alpha z \\
\text { et }|\operatorname{Arg} \alpha| \geq \frac{\pi}{4}
\end{array}\right\} .
$$

On vérifie sans peine que $A$ est un ensemble semi-analytique. Supposons que la Proposition 2 ne soit pas vérifiée c'est-à-dire que 0 soit adhérent à $A$. D'après le Lemme 2 , il existe un chemin analytique réel $P:[0, r) \rightarrow$ $\mathbb{C}^{n}$ tel que:

(1) $P(0)=0$

(2) $P(t) \in A$ pour tout $t$ strictement positif, i.e.

$$
\left\{\begin{array}{l}
P(t) \notin X_{0} \\
\sum_{k=1}^{m} \overline{\lambda_{k}} \operatorname{grad} \log f_{k}(P(t))=\alpha(t) P(t) \\
|\operatorname{Arg} \alpha(t)| \geq \frac{\pi}{4} .
\end{array}\right.
$$

ce qui entraine d'après le Lemme 1 que

$$
\lim _{t \rightarrow 0} \operatorname{Arg} \alpha(t)=0
$$

ce qui est contradictoire avec $|\operatorname{Arg} \alpha(t)| \geq \frac{\pi}{4}$. Et la Proposition 2 est démontrée.

\section{Proposition 3.}

Pour $\varepsilon$ réel strictement positif assez petit, il existe sur $\varepsilon B^{2 n} \backslash X_{0}$ un champ de vecteurs $\chi, C^{\infty}$, tel que pour tout $z \in \varepsilon B^{2 n} \backslash X_{0}$ on ait:

(1) la partie réelle de $\langle\chi(z), z\rangle$ est un réel strictement positif.

(2) $\left\langle\chi(z), \sum_{k=1}^{m} \overline{\lambda_{k}} \operatorname{grad} \log f_{k}(z)\right\rangle$ est un réel strictement positif. 
Démonstration de la Proposition 3:

L'idée est la suivante:

On construit le champ de vecteurs, $\chi$, localement puis on recolle avec un partition de l'unité.

D'après la Proposition 2, il existe $\varepsilon_{0}$, réel strictement positif tel que pour tout $z \in \varepsilon B^{2 n} \backslash X_{0}, 0<\varepsilon \leq \varepsilon_{0}$, les vecteurs $z$ et $\sum_{k=1}^{m} \overline{\lambda_{k}} \operatorname{grad} \log f_{k}(z)$ sont ou bien linéairement indépendants sur $\mathbb{C}$ ou bien on a

$$
\sum_{k=1}^{m} \overline{\lambda_{k}} \operatorname{grad} \log f_{k}(z)=\alpha z
$$

avec $\alpha \in \mathbb{C} \backslash\{0\}$ et $|\operatorname{Arg} \alpha|<\frac{\pi}{4}$.

Nous distinguons les deux cas suivants:

1er Cas: si $\sum_{k=1}^{m} \overline{\lambda_{k}} \operatorname{grad} \log f_{k}(z)=\alpha z$ avec $\alpha \in \mathbb{C} \backslash\{0\}$ et $|\operatorname{Arg} \alpha|<$ $\frac{\pi}{4}$, on prend $\chi(z)=\sum_{k=1}^{m} \overline{\lambda_{k}} \operatorname{grad} \log f_{k}(z)$.

On a:

$\operatorname{Re}\langle\chi(z), z\rangle=\operatorname{Re}\langle\alpha z, z\rangle=\operatorname{Re} \alpha\|z\|^{2}$ est strictement positif. De plus: $\left\langle\chi(z), \sum_{k=1}^{m} \overline{\lambda_{k}} \operatorname{grad} \log f_{k}(z)\right\rangle=\langle\alpha z, \alpha z\rangle=|\alpha|^{2}\|z\|^{2}$ est un réel strictement positif.

2ème Cas: si $z$ et $\sum_{k=1}^{m} \overline{\lambda_{k}} \operatorname{grad} \log f_{k}(z)$ sont linéairement indépendants sur $\mathbb{C}$ alors $z, i z, \sum_{k=1}^{m} \overline{\lambda_{k}} \operatorname{grad} \log f_{k}(z)$ et $i \sum_{k=1}^{m} \overline{\lambda_{k}} \operatorname{grad} \log f_{k}(z)$ sont linéairement indépendants sur $\mathbb{R}$. D'autre part nous avons l'équivalence:

$$
\left\langle\chi(z), \sum_{k=1}^{m} \overline{\lambda_{k}} \operatorname{grad} \log f_{k}(z)\right\rangle
$$

est un réel strictement positif si et seulement si:

$$
\left\{\begin{array}{l}
\operatorname{Re}\left\langle\chi(z), \sum_{k=1}^{m} \overline{\lambda_{k}} \operatorname{grad} \log f_{k}(z)\right\rangle>0 \\
\operatorname{Im}\left\langle\chi(z), \sum_{k=1}^{m} \overline{\lambda_{k}} \operatorname{grad} \log f_{k}(z)\right\rangle=0
\end{array}\right.
$$


or

$\operatorname{Im}\left\langle\chi(z), \sum_{k=1}^{m} \overline{\lambda_{k}} \operatorname{grad} \log f_{k}(z)\right\rangle=\operatorname{Re}\left\langle\chi(z), i \sum_{k=1}^{m} \overline{\lambda_{k}} \operatorname{grad} \log f_{k}(z)\right\rangle$.

Donc on doit chercher un vecteur $\chi(z)$ tel que:

$$
\left\{\begin{array}{l}
\operatorname{Re}\langle\chi(z), z\rangle>0 \\
\operatorname{Re}\left\langle\chi(z), \sum_{k=1}^{m} \overline{\lambda_{k}} \operatorname{grad} \log f_{k}(z)\right\rangle>0 \\
\operatorname{Re}\left\langle\chi(z), i \sum_{k=1}^{m} \overline{\lambda_{k}} \operatorname{grad} \log f_{k}(z)\right\rangle=0
\end{array}\right.
$$

Etant donné l'indépendance linéaire réelle des quatres vecteurs $z, i z$, $\sum_{k=1}^{m} \overline{\lambda_{k}} \operatorname{grad} \log f_{k}(z)$ et $i \sum_{k=1}^{m} \overline{\lambda_{k}} \operatorname{grad} \log f_{k}(z)$, il est toujours possible de trouver un tel vecteur $\chi(z)$.

\section{Proposition 4.}

Les trajectoires du champ de vecteurs, $\chi$, précédent vérifient les propriétés suivantes:

si $t \rightarrow \varphi(t)$ est une trajectoire de $\chi$ alors:

(1) la fonction: $t \rightarrow\|\varphi(t)\|$ est strictement croissante.

(2) la fonction: $t \rightarrow \sum_{k=1}^{m}\left[\left(\operatorname{Im} \lambda_{k}\right) \log \left|f_{k}(\varphi(t))\right|+\operatorname{Re} \lambda_{k} \operatorname{Arg}\left(f_{k}(\varphi(t))\right)\right]$ est constante i.e. que le champ de vecteurs $\chi$ est tangent aux feuilles du feuilletage défini par la forme de Pfaff $\Omega$.

(3) la fonction $t \rightarrow \mid F(\varphi(t) \mid$ est strictement croissante.

Démonstration de la Proposition 4:

Soit $t \rightarrow \varphi(t)$ une trajectoire de $\chi$, on a:

$$
\frac{d \varphi(t)}{d t}=\chi(\varphi(t))
$$

D'après la Remarque 1et la Remarque 2 , on a pour tout $k$ compris entre 1 et $m$ :

$$
\frac{d}{d t}\left(f_{k}(\varphi(t))\right)=\left\langle\frac{d(\varphi(t))}{d t}, \overline{f_{k}(\varphi(t))} \operatorname{grad} \log f_{k}(\varphi(t))\right\rangle .
$$


Soit:

$$
\frac{1}{f_{k}(\varphi(t))} \frac{d}{d t}\left(f_{k}(\varphi(t))\right)=\left\langle\frac{d \varphi(t)}{d t}, \operatorname{grad} \log f_{k}(\varphi(t))\right\rangle
$$

et par conséquent:

(*) $\frac{d}{d t}\left(\sum_{k=1}^{m} \lambda_{k} \log f_{k}(\varphi(t))\right)=\left\langle\frac{d \varphi(t)}{d t}, \sum_{k=1}^{m} \overline{\lambda_{k}} \operatorname{grad} \log f_{k}(\varphi(t))\right\rangle$

or:

$$
\begin{aligned}
\sum_{k=1}^{m} \lambda_{k} \log f_{k}(\varphi(t))= & \left.\left.\log \left(\mid f_{1}(\varphi(t)) \cdot f_{2}{ }^{\lambda_{1}} \varphi(t)\right) \ldots f_{m} \stackrel{\lambda_{m}}{(} \varphi(t)\right) \mid\right) \\
& \left.\left.+i \operatorname{Arg}\left(f_{1}^{\lambda_{1}}(\varphi(t)) \cdot f_{2} \stackrel{\lambda_{2}}{(} \varphi(t)\right) \ldots f_{m} \stackrel{\lambda_{m}}{(} \varphi(t)\right)\right) .
\end{aligned}
$$

En remplaçant dans $(*)$ on obtient:

$$
\begin{aligned}
\left.\frac{d}{d t} \log \left(\mid f_{1}(\varphi(t)) \cdot f_{2}(\varphi(t)) \ldots f_{m} \stackrel{\lambda_{2}}{\left(\lambda_{m}\right.} \varphi(t)\right) \mid\right) \\
+i \frac{d}{d t} \operatorname{Arg}\left(f_{1}(\varphi(t)) \cdot f_{2}{ }^{\lambda_{1}}(\varphi(t)) \ldots f_{m}(\varphi(t))\right) \\
=\left\langle\chi(\varphi(t)), \sum_{k=1}^{\lambda_{m}} \overline{\lambda_{k}} \operatorname{grad} \log \left(f_{k}(\varphi(t))\right\rangle .\right.
\end{aligned}
$$

D'après la Proposition $3,\left\langle\chi(\varphi(t)), \sum_{k=1}^{m} \overline{\lambda_{k}} \operatorname{grad} \log \left(f_{k}(\varphi(t))\right)\right\rangle$ est un réel strictement positif donc:

$$
\left\{\begin{array}{l}
\frac{d}{d t} \log \left(\left|f_{1}(\varphi(t)) \cdot f_{2}{ }^{\lambda_{1}}(\varphi(t)) \ldots f_{m}(\varphi(t))\right|\right)>0 \\
\frac{d}{d t} \operatorname{Arg}\left(f_{1}(\varphi(t)) \cdot f_{2}^{\lambda_{1}}(\varphi(t)) \ldots f_{m}{ }^{\lambda_{2}}(\varphi(t))\right)=0 .
\end{array}\right.
$$

Ce qui implique, d'une part que la fonction:

$$
\left.\left.t \rightarrow \mid f_{1}(\varphi(t)) \cdot f_{2} \stackrel{\lambda_{2}}{\left(\lambda_{1}\right.} \varphi(t)\right) \ldots f_{m} \stackrel{\lambda_{m}}{(} \varphi(t)\right) \mid
$$

est strictement croissante et d'autre part que la fonction:

$$
t \rightarrow \sum_{k=1}^{m}\left[\left(\operatorname{Im} \lambda_{k}\right) \log \left|f_{k}(\varphi(t))\right|+\left(\operatorname{Re} \lambda_{k}\right) \operatorname{Arg} f_{k}(\varphi(t))\right]
$$


est constante.

Il reste à prouver que la fonction:

$$
t \rightarrow\|\varphi(t)\|
$$

est strictement croissante.

On a:

$$
\frac{d}{d t}\|\varphi(t)\|^{2}=\frac{d}{d t}\langle\varphi(t), \varphi(t)\rangle=\operatorname{Re}\left\langle\frac{d \varphi(t)}{d t}, \varphi(t)\right\rangle=\operatorname{Re}\langle\chi(\varphi(t)), \varphi(t)\rangle
$$

D'après la Proposition $3, \operatorname{Re}\langle\chi(\varphi(t)), \varphi(t)\rangle$ est strictement positif donc la fonction: $t \rightarrow\|\varphi(t)\|$ est strictement croissante. Ce qui achève la démonstration.

\section{Remarque 4.}

Les intégrales de la forme de Pfaff $\omega$ (respectivement $\Omega$ ) sont les "hypersurfaces de niveau" de la fonction multiforme $F$ (respectivement $\operatorname{Arg} F)$.

Pour $\alpha \in \mathbb{C}$, désignons par $H_{\alpha}$ (respectivement $G_{\alpha}$ ) "l'hypersurface de niveau $\alpha$ " de la fonction multiforme $F$ (respectivement de la fonction multiforme $\operatorname{Arg} F$ ).

Nous avons la:

\section{Proposition 5.}

Il existe $\eta_{0}$ réel strictement positif tel que pour tout $\eta, 0<\eta \leq \eta_{0}$, $i l$ existe un $C^{\infty}$ difféormorphisme $\Psi$ de $\left(\varepsilon S^{2 n-1} \backslash \varepsilon K\right) \cap[|F|>\eta]$ sur $\varepsilon S^{2 n-1} \backslash \varepsilon K$ tel que pour tout $\alpha \in \mathbb{C} \backslash\{0\}$, on ait:

$$
\Psi\left(G_{\alpha} \cap\left(\varepsilon S^{2 n-1} \backslash \varepsilon K\right) \cap[|F|>\eta]\right)=G_{\alpha} \cap\left(\varepsilon S^{2 n-1} \backslash \varepsilon K\right)
$$

Démonstration de la Proposition 5:

Désignons par $g$ la fonction de $\varepsilon S^{2 n-1} \backslash \varepsilon K$ à valeurs réelles définies par:

$$
g(z)=-\log (|F(z)|)
$$

et par $g_{\alpha}$ la restriction de $g$ à $G_{\alpha}$ pour $\alpha \in \mathbb{C} \backslash\{0\}$.

Nous avons le résultat suivant: 


\section{Lemme 3.}

Il existe $\delta_{\alpha}$ réel strictement positif tel que tous les points critiques de $g_{\alpha}$ sont contenus dans le compact $C_{\alpha}$ suivant:

$$
C_{\alpha}=\left\{z \in G_{\alpha}:|F(z)| \geq \delta_{\alpha}\right\} .
$$

De même il existe $\delta$ réel strictement positif tel que tous les points critiques de $g$ sont contenus dans le compact $C$ suivant:

$$
C=\left\{z \in \varepsilon S^{2 n-1} \backslash \varepsilon K:|F(z)| \geq \delta\right\} .
$$

Démonstration du Lemme 3:

Nous allons démontrer d'abord deux propositions à partir desquelles nous déduirons le Lemme 3 .

Pour $\varepsilon$ assez petit et $z \in \varepsilon S^{2 n-1} \backslash X_{0}$ désignons par $T_{z} G_{\alpha}$ l'espace tangent à $G_{\alpha}$ en $z$. $T_{z} G_{\alpha}$ est un espace vectoriel sur $\mathbb{R}$ de dimension $2 n-2$. Notons $N_{z} G_{\alpha}$ l'espace normal à $G_{\alpha}$ en $z$.

$N_{z} G_{\alpha}=\left\{v \in \mathbb{C}^{n}\right.$ tel que $\operatorname{Re}\langle u, v\rangle=0$ quelque soit $\left.u \in T_{z} G_{\alpha}\right\}$.

$N_{z} G_{\alpha}$ est un espace vectoriel sur $\mathbb{R}$ de dimension 2.

\section{Lemme 4.}

Pour $\varepsilon$ suffisamment petit et $z \in \varepsilon S^{2 n-1} \backslash X_{0}, N_{z} G_{\alpha}$ est le sous-espace vectoriel engendré par $z$ et $i \sum_{k=1}^{m} \overline{\lambda_{k}} \operatorname{grad} \log f_{k}(z)$.

Démonstration du Lemme 4:

Vérifions d'abord que: $\sum_{k=1}^{m} \overline{\lambda_{k}} \operatorname{grad} \log f_{k}(z) \in N_{z} G_{\alpha}$ et que $z \in$ $N_{z} G_{\alpha}$.

Soit $t \rightarrow P(t)$ un chemin analytique dans $G_{\alpha}$ tel que $P(0)=z$ et $P^{\prime}(0) \in T_{z} G_{\alpha}$.

*On a d'une part:

$$
\operatorname{Arg} F(P(t))=\alpha
$$

donc:

$$
\begin{aligned}
\operatorname{Re}\left\langle P^{\prime}(0), i \sum_{k=1}^{m} \overline{\lambda_{k}} \operatorname{grad} \log f_{k}(P(t))\right\rangle & =\operatorname{Re}\left(-i \frac{d}{d t}\left(\sum_{k=1}^{m} \lambda_{k} \log f_{k}(P(t))\right)\right) \\
& =\frac{d}{d t}(\operatorname{Arg}(F(P(t))))=\frac{d}{d t} \alpha=0
\end{aligned}
$$


c'est à dire $\sum_{k=1}^{m} \overline{\lambda_{k}} \operatorname{grad} \log f_{k}(z) \in N_{z} G_{\alpha}$.

*On a d'autre part:

$$
\operatorname{Re}\left\langle\frac{d P(t)}{d t}, P(t)\right\rangle=\frac{1}{2} \frac{d}{d t}\|P(t)\|^{2}
$$

ce qui entraine $\operatorname{Re}\left\langle P^{\prime}(0), z\right\rangle=0$ (car $P(0)=z$ et $\left\|z^{2}\right\|=\varepsilon^{2}$ puisque $\left.z \in \varepsilon S^{2 n-1} \backslash X_{0}\right)$ c'est à dire $z \in N_{z} G_{\alpha}$.

Or on sait d'après la Proposition 1 que $z$ et $i \sum_{k=1}^{m} \overline{\lambda_{k}} \operatorname{grad} \log f_{k}(z)$ sont linéairement indépendants sur $\mathbb{R}$ donc ils forment une base de $N_{z} G_{\alpha}$. D'où le résultat.

\section{Lemme 5 .}

Désignant par $\Sigma_{\alpha}$ (respectivement $\Sigma$ ) l'ensemble des points critiques de $g_{\alpha}$ (respectivement de $g$ ) on a:

$$
\begin{aligned}
\Sigma_{\alpha} & =\left\{z \in G_{\alpha} \text { tel que: } \sum_{k=1}^{m} \overline{\lambda_{k}} \operatorname{grad} \log f_{k}(z)=\theta z, \theta \in \mathbb{C}\right\} \\
(\operatorname{resp} . \Sigma & \left.=\left\{z \in \varepsilon S^{2 n-1} \backslash \varepsilon K: \sum_{k=1}^{m} \overline{\lambda_{k}} \operatorname{grad} \log f_{k}(z)=\theta z, \theta \in \mathbb{C}\right\}\right) .
\end{aligned}
$$

Démonstration du Lemme 5:

Soit $0<\varepsilon \leq \varepsilon_{0}\left(\varepsilon_{0}\right.$ étant celui de la Proposition 1) et soit $z \in \varepsilon S^{2 n-1} \backslash$ $\varepsilon K$. On a les équivalences suivantes:

$$
\begin{aligned}
& z \text { est un point critique pour } g_{\alpha} \\
& \Longleftrightarrow {\left[\begin{array}{l}
\text { Pour tout } P: t \rightarrow P(t) \text { un chemin analytique contenu } \\
\text { dans } G_{\alpha} \text { tel que } P(0)=z \text { on a: } \\
\frac{d}{d t}\left(g_{\alpha}(P(t))\right)_{\mid t=0}=0
\end{array}\right.} \\
& \Longleftrightarrow \operatorname{Re}\left\langle P^{\prime}(0), \sum_{k=1}^{m} \overline{\lambda_{k}} \operatorname{grad} \log f_{k}(P(t))\right\rangle=0 \\
& \Longleftrightarrow \sum_{k=1}^{m} \overline{\lambda_{k}} \operatorname{grad} \log f_{k}(P(t)) \in N_{z} G_{\alpha}
\end{aligned}
$$


$\Longleftrightarrow$ Il existe $r, s \in \mathbb{R}$ tel que:

$$
\sum_{k=1}^{m} \overline{\lambda_{k}} \operatorname{grad} \log f_{k}(z)=r z+s i \sum_{k=1}^{m} \overline{\lambda_{k}} \operatorname{grad} \log f_{k}(z)
$$

$\Longleftrightarrow$ Il existe $r, s \in \mathbb{R}$ tel que: $\sum_{k=1}^{m} \overline{\lambda_{k}} \operatorname{grad} \log f_{k}(z)=\frac{r}{1-i s} \cdot z$

$\Longleftrightarrow$ Il existe $\theta \in \mathbb{C}$ tel que: $\sum_{k=1}^{m} \overline{\lambda_{k}} \operatorname{grad} \log f_{k}(z)=\theta z$.

Un raisonnement analogue permet de démontrer la deuxième partie de la Proposition 7.

Déduisons maintenant le Lemme 3 à partir des Lemmes 4 et 5 . Raisonnons par l'absurde. Supposons donc qu'il existe une suite

$$
\left(Z_{n}\right)_{n \geq 1}, Z_{n} \in \sum_{\alpha} \text { tel que }\left|F\left(Z_{n}\right)\right|<\frac{1}{n} .
$$

La suite $\left(Z_{n}\right)_{n \geq 1}$ tend vers une limite $a$ dans le compact $\varepsilon S^{2 n-1}$ donc le point $a$ est adhérent à $\Sigma_{\alpha}$ et par conséquent d'après le Lemme 2, il existe un chemin analytique $P:[0, r) \rightarrow \Sigma_{\alpha}$ tel que:

(1) $P(0)=a$

(2) $P(t) \in \Sigma_{\alpha}$ pour tout $t$ strictement positif.

Soit $t \in] 0, r[$, on a:

$$
\frac{d}{d t} g_{\alpha}(P(t))=\left\langle P^{\prime}(0), \operatorname{grad} g_{\alpha}(P(t))\right\rangle=0
$$

donc pour tout $t \in] 0, r[, P(t)$ est un point critique pour la fonction $z \rightarrow|F(z)|$ ce qui entraîne que:

$$
|F(P(t))|=\text { cste pour tout } t \in] 0, r[.
$$

Par continuité en faisant tendre $t$ vers zéro on obtient:

$$
|F(P(t))|=0 \text { pour tout } t \in] 0, r[
$$

ce qui entraine que pour tout $t$ strictement positif $P(t) \in X_{0}$, or:

$$
P(t) \in \sum_{\alpha} \subset G_{\alpha} \subset \varepsilon S^{2 n-1} \backslash X_{0} .
$$

D'où la contradiction.

Un raisonnement analogue permet de prouver la deuxième partie du Lemme 3. 
Lemme 6 (cf. [3]).

Soit $h: M \rightarrow \mathbb{R}$ une fonction $C^{\infty}$ d'une variété lisse $M$ à valeurs réelles. Pour $r \in \mathbb{R}$ notons $M^{r}$ l'ensemble suivant:

$$
M^{r}=h^{-1}(-\infty, r]=\{P \in M \text { tel que: } h(P) \leq r\} .
$$

Soient $r_{1}, r_{2}$ deux nombres réels avec $r_{1}<r_{2}$ tels que:

$$
h^{-1}\left[r_{1}, r_{2}\right]=\left\{P \in M \text { tel que: } r_{1} \leq h(P) \leq r_{2}\right\}
$$

est un compact et ne contient aucun point critique de $h$, alors: il existe un $C^{\infty}$ difféomorphime $\Phi: M \rightarrow M$ tel que

$$
\Phi\left(M^{r_{1}}\right)=M^{r_{2}} .
$$

Démonstration du Lemme 6:

Nous ne donnons ici qu'une esquisse de démonstration car nous aurons besoin dans la suite de savoir comment est construit le difféormorphisme $\Phi$. Quand aux détails de la démonstration, ils se trouvent dans [3].

L'idée de la démonstration est de pousser $M^{r_{1}}$ sur $M^{r_{2}}$ avec un champ de vecteurs transverse aux hypersurfaces $h=$ cste.

Choissisons une métrique Riemanniène sur $M$ et notons $\langle$,$\rangle le produit$ hermitien déterminé par cette métrique.

Soit $v: M \rightarrow \mathbb{R}$ une fonction $C^{\infty}$ qui est égale à $\frac{1}{\langle\operatorname{grad} h, \operatorname{grad} h\rangle}$ sur le compact $h^{-1}\left[r_{1}, r_{2}\right]$ et qui s'annule en dehors d'un voisinage compact de $h^{-1}\left[r_{1}, r_{2}\right]$.

Considérons le champ de vecteurs $\Delta$ défini par:

$$
\Delta p=v(p)(\operatorname{grad} h)_{p}, \text { pour } p \in M .
$$

$\Delta$ engendre un groupe un paramètre de difféomorphismes

$$
\varphi_{t}: M \rightarrow M .
$$

Pour $p \in M$ si $\varphi_{t}(p) \in h^{-1}\left[r_{1}, r_{2}\right]$ alors $\frac{d h}{d t}\left(\varphi_{t}(p)\right)=1$.

Le difféomorphisme $\varphi_{r_{2}-r_{1}}: M \rightarrow M$ envoie difféomorphiquement $M^{r_{1}}$ sur $M^{r_{2}}$.

Déduisons maintenant la démonstration de la Proposition 5 à partir des résultats précédents.

D'après le Lemme 3 , il existe $\delta$ (respectivement $\delta_{\alpha}$ ) strictement positif tel que tous les points critiques de la fonction $g$ (respectivement $g_{\alpha}$ ) sont contenus dans le compact $C$ (respectivement $C_{\alpha}$ ). 
Considérons la variété $M$ suivante:

$$
M=\left\{z \in \varepsilon S^{2 n-1} \backslash \varepsilon K \text { tel que: }|F(z)|<\delta\right\}
$$

$M$ ne contient aucun point critique de la fonction $g: z \rightarrow-\log |F(z)|$, et par conséquent $M$ ne contient aucun point critique de la fonction $z \rightarrow \frac{1}{|F(z)|}$.

Posons $\eta_{0}=\delta$ et soient $\rho$ et $\eta$ deux nombres réels strictement positifs tel que: $0<\rho<\eta \leqslant \eta_{0}$. L'ensemble

$$
\left\{z \in \varepsilon S_{2 n-1} \backslash \varepsilon K \text { tel que } \frac{1}{\eta} \leq \frac{1}{|F(z)|} \leq \frac{1}{\rho}\right\}
$$

est un compact de $M$ et ne contient aucun point critique de la fonction $\frac{1}{|F|}: M \rightarrow \mathbb{R}$. Donc d'après le Lemme 6 , il existe un $C^{\infty}$ difféomorphisme $\Psi$ de

$$
\left\{z \in \varepsilon S^{2 n-1} \backslash \varepsilon K \text { tel que: } \frac{1}{|F(z)|}<\frac{1}{\eta}\right\}
$$

sur

$$
\left\{z \in \varepsilon S^{2 n-1} \backslash \varepsilon K \text { tel que: } \frac{1}{|F(z)|}<\frac{1}{\rho}\right\}
$$

c'est-à -dire un $C^{\infty}$ difféomorphisme de

$$
\left\{z \in \varepsilon S^{2 n-1} \backslash \varepsilon K \text { tel que: }|F(z)|>\eta\right\}
$$

sur

$$
\left\{z \in \varepsilon S^{2 n-1} \backslash \varepsilon K \text { tel que }|F(z)|>\rho\right\} .
$$

Comme $\rho$ est arbitraire, nous en déduisons qu'il existe un $C^{\infty}$ difféomorphisme, que nous noterons encore $\psi$, de

$$
\left\{z \in \varepsilon S^{2 n-1} \backslash \varepsilon K \text { tel que: }|F(z)|>\eta\right\} \text { sur } \varepsilon S^{2 n-1} \backslash \varepsilon K .
$$

En considérant la variété $M_{\alpha}$ suivante:

$$
M_{\alpha}=\left\{z \in G_{\alpha} \cap\left(\varepsilon S^{2 n-1} \backslash \varepsilon K\right) \text { tel que: }|F(z)|<\delta_{\alpha}\right\}
$$

et la fonction $g_{\alpha}$, on démontre de façon tout à fait analogue qu'il existe un $C^{\infty}$ difféomorphisme $\Psi_{\alpha}$ de

$$
\left\{z \in G_{\alpha} \cap\left(\varepsilon S^{2 n-1} \backslash \varepsilon K\right) \text { tel que: }|F(z)|>\eta_{\alpha}\right\}
$$

sur

$$
G_{\alpha} \cap\left(\varepsilon S^{2 n-1} \backslash \varepsilon K\right) .
$$


De plus par construction de $\Psi$, en fonction de $g$, ainsi que de $\Psi_{\alpha}$ en fonction de $g_{\alpha}$, il est clair que $\Psi_{\alpha}$ est la restriction de $\Psi$ à $M_{\alpha}$.

En résumé, il existe un $C^{\infty}$ difféomorphisme $\Psi$ de

$$
\left\{z \in\left(\varepsilon S^{2 n-1} \backslash \varepsilon K\right) \text { tel que: }|F(z)|>\eta_{\alpha}\right\}
$$

sur

$$
\varepsilon S^{2 n-1} \backslash \varepsilon K
$$

tel que:

$$
\begin{aligned}
\Psi\left(G_{\alpha} \cap\left(\varepsilon S^{2 n-1} \backslash \varepsilon K\right) \cap[|F|>\eta]\right) & =\Psi_{\alpha}\left(G_{\alpha} \cap\left(\varepsilon S^{2 n-1} \backslash \varepsilon K\right) \cap\left[|F|>\eta_{\alpha}\right]\right) \\
& =G_{\alpha} \cap\left(\varepsilon S^{2 n-1} \backslash \varepsilon K\right) .
\end{aligned}
$$

Et la Proposition 5 est démontrée.

Nous sommes maintenant en mesure de prouver le théorème à partir des résultats précédents.

Soient $\varepsilon$ et $\eta$ deux nombres réels strictements positifs tels que: $0<$ $\varepsilon \leq \varepsilon_{0}$ et $0<\eta \leq \eta_{0}$ avec $\varepsilon_{0}$ et $\eta_{0}$ suffisamment petits.

Soit $\beta \in \mathbb{C} \backslash\{0\}$ et $H_{\beta}$ "l'hypersurface de niveau $\beta$ " de la fonction multiforme $F$. Il existe sur $\varepsilon B^{2 n} \backslash X_{0}$ un champ de vecteurs $\chi, C^{\infty}$, tangent aux feuilles du feuilletage défini par la forme de pfaff $\Omega$ et dont le flot $\Phi$ en voie de façon $C^{\infty}$ difféomorphe:

$$
H_{\beta} \cap[|F|=\eta] \cap \varepsilon B^{2 n}
$$

sur

$$
G_{\alpha} \cap\left(\varepsilon S^{2 n-1} \backslash \varepsilon K\right) \cap[|F|>\eta]
$$

où $\alpha$ est tel que:

$$
\beta=\eta e^{i \alpha} .
$$

D'autre part, il existe un $C^{\infty}$ difféomorphisme $\Psi$ de $\left(\varepsilon S^{2 n-1} \backslash \varepsilon K\right) \cap$ $[|F|>\eta]$ sur $\varepsilon S^{2 n-1} \backslash \varepsilon K$ qui envoie

$$
G_{\alpha} \cap\left(\varepsilon S^{2 n-1} \backslash \varepsilon K\right) \cap[|F|>\eta]
$$

sur

$$
G_{\alpha} \cap\left(\varepsilon S^{2 n-1} \backslash \varepsilon K\right)
$$

de façon $C^{\infty}$ difféomorphe, d'où le résultat. 


\section{References}

1. A. KaBILA, Un théorème de fibration à la Milnor pour les germes de formes de Pfaff logarithmiques, C. R. Acad. Sci. Paris Sér. I. 302 (1986), 511-514.

2. J. Milnor, "Singular points of complex hypersurfaces," Ann. Math. Studies 61, Princeton University Press, 1968.

3. J. Milnor, "Morse theory," Ann. Math. Studies 51, Princeton University Press, 1963.

4. R. NARASImHAN, "Introduction to the theory of analytic spaces," Lectures Notes in Math. 25, Springer-Verlag, 1966.

I.S.T.V.-Département de Mathématiques

URA au CNRS D-751

Université de Valenciennes et du Hainaut Cambrésis

59304 Valenciennes cedex

FRANCE

Primera versió rebuda el 25 d'Abril de 1995 , darrera versió rebuda el 6 de Novembre de 1995 